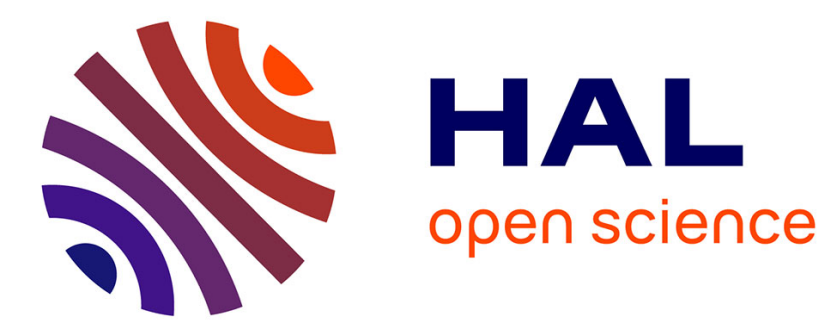

\title{
Could omega-3 fatty acids prevent rheumatoid arthritis?
}

Olfa Saidane, Luca Semerano, Jérémie Sellam

\section{To cite this version:}

Olfa Saidane, Luca Semerano, Jérémie Sellam. Could omega-3 fatty acids prevent rheumatoid arthritis?. Joint Bone Spine, 2018, 85 (June 2018), 10.1016/j.jbspin.2018.05.007 . hal-01955933

\section{HAL Id: hal-01955933 https://hal.sorbonne-universite.fr/hal-01955933}

Submitted on 14 Dec 2018

HAL is a multi-disciplinary open access archive for the deposit and dissemination of scientific research documents, whether they are published or not. The documents may come from teaching and research institutions in France or abroad, or from public or private research centers.
L'archive ouverte pluridisciplinaire HAL, est destinée au dépôt et à la diffusion de documents scientifiques de niveau recherche, publiés ou non, émanant des établissements d'enseignement et de recherche français ou étrangers, des laboratoires publics ou privés. 


\section{Could omega-3 fatty acids prevent rheumatoid arthritis?}

\section{Olfa SAIDANE ${ }^{1,2}$, Luca SEMERANO ${ }^{3,4,5}$, Jérémie SELLAM ${ }^{6,7,8,9}$}

${ }^{1}$ Service de rhumatologie, Hôpital Charles Nicolle, Faculté de Médecine de Tunis, Tunisie

${ }^{2}$ University of Tunis El Manar, Tunisia

${ }^{3}$ Service de rhumatologie, groupe hospitalier Avicenne, Jean-Verdier-René-Muret, Assistance publique-hôpitaux de Paris (AP-HP), Bobigny, France

${ }^{4}$ Inserm UMR 1125, Bobigny, France

${ }^{5}$ Université Paris 13, Sorbonne-Paris, Bobigny France

${ }^{6}$ Sorbonne Université, UPMC Univ Paris 06, Paris, France

7 INSERM UMRS_938, CRSA, Paris, France

${ }^{8}$ Département Hospitalo-Universitaire "Inflammation-Immunopathologie-Biotherapie" (DHU i2B), Paris, France

${ }^{9}$ Service de rhumatologie, hôpital Saint-Antoine, AP-HP, Paris, France

Keywords: Rheumatoid arthritis, omega-3, n-3 polyunsaturated fatty acids, diet, prevention, inflammation

Number of words: 1639

Number of pages: 10

Number of Figure: 1

\section{Corresponding author}

Pr Jérémie SELLAM, Department of Rheumatology, Assistance Publique-Hôpitaux de Paris (AP-HP), Saint-Antoine Hospital, 184 rue du Faubourg Saint-Antoine, 75012, Paris, France. E-mail : jeremie.sellam@aphp.fr Phone: +3314928 2520

Fax +33 149282513

\section{Disclosure of interest: none}


Rheumatoid arthritis (RA) is a systemic inflammatory disease in which both genetics and environment play a role. Diet is an environmental factor that has been studied in other inflammatory conditions [1-3]. Particularly, omega-3, also called n-3 polyunsaturated fatty acids (n3PUFAs); essential fatty acids (omega-3 FA) have been found to have a suppressive action on inflammation and are thought to modulate immune response [4-6]. New data showed that omega-3 FA may also interfere in the pathophysiology of rheumatoid arthritis, namely in the steps that lead from the preclinical phase to established disease, which may open new perspectives for the prevention of this disease.

\section{Mechanisms of action of omega-3 fatty acids: anti-inflammatory and immunomodulatory effects}

Different mechanisms of action could explain the effects of omega-3 FA in the prevention of RA. Omega-3 FA have been shown to inhibit the inflammatory process by decreasing adhesion molecule expression, reducing chemotactic response of leucocytes and inducing the production of the anti-inflammatory lipid mediators protectins and resolvins [7]. They are also known to affect cytokine production, alter T-cell reactivity and proliferation [8] and to modulate Th1/Th2 balance [5]. Some of these effects can be explained by a change in the composition of inflammatory cell membrane phospholipids induced by incorporating omega-3 FA at the expense of arachidonic acid [7]. They can inhibit the expression of major histocompatibility complex class II molecules and thus alter antigen presentation function [9]. More recently, one omega-3 FA, docosahexaenoic acid (DHA), has been identified as a powerful activator of the AMP-activated protein kinase/Sirtuine 1 pathway, which inhibits NF- kappaB-p65 and acts to redirect immune cells towards an immunoregulatory phenotype [10]. Overall, both innate and immune adaptive 
responses seem to be affected by omega-3 FA. Thus, it seems relevant to investigate their role in the pathophysiology of RA throughout its different phases, from genetic predisposition, to pre-symptomatic autoimmunity, up to the established disease [Figure 1].

\section{Omega-3 fatty acids in experimental models of arthritis}

Experimental models have highlighted the immunomodulatory activity of omega-3 FA. In rat collagen induced arthritis ( $\mathrm{CIA})$, the preventive administration of a rich source of the omega-3 FA alpha linolenic acid (ALA), the Linum usitatissimum fixed oil (Linseed Oil), resulted in a significant reduction of joint swelling and lower serum TNF alpha levels [11]. Similar findings were reported in a study in which a diet supplemented with eicosapentaenoic acid (EPA) and DHA (contained in krill oil) significantly inhibited the development of arthritis in DBA/1 mice CIA [12]. In this study, the preventive supplementation with krill oil, but not with fish oil, reduced inflammatory cell infiltration of the synovial membrane and led to a lower erosion score. Both diets led to significantly lower synovial hyperplasia score when compared with the control group. Krill oil and fish oil are particularly balanced in EPA and DHA. However, krill oil is different in that it contains a majority of omega-3 fatty acids in the form of phospholipids, which may possibly result in better bioavailability [13].

\section{Omega-3 fatty acids and the incidence of RA in epidemiological studies}

The possible preventive effect of omega-3 FA on the incidence of RA was investigated in several observational studies. All evaluated fish and oil fish intake based on food frequency questionnaires [14-16]. Overall results were conflicting. 
A large cohort study showed that daily intake of fat fish was protective against RA whereas medium fat fish consumption was associated with an increased risk [14]. Nested case-control studies conducted within large cohorts compared newly diagnosed RA cases with controls for their fish consumption up to 5 years before disease clinical onset. The study conducted by Rosell et al. on the Swedish cohort EIRA was inconclusive [15], likely because of high background consumption of fatty fish in Scandinavian populations. Conversely, in a US cohort, even moderate fish consumption was associated with a decreased risk of RA (adjusted odds ratio (OR): $0.57 ; 95 \%$ confidence interval $(95 \% \mathrm{Cl}): 0.35-0.93)$ [16]. Interestingly, in the latter study, the association became stronger when the analysis was restricted to rheumatoid factor positive patients.

More recently, a nation-wide Swedish prospective cohort study assessed long chain omega-3 FA dietary intake (calculated by the frequency and the nutrient content of portions) and the risk of developing RA among women aged 54 and older [17]. After adjustment for other risk factors, long chain omega-3 FA consumption was associated to a lower risk of developing RA (relative risk: $0.65 ; 95 \% \mathrm{Cl}: 0.48-0.9$ ). Long term intake during 10 years was more strongly protective.

The largest study was nested in the Nurse's Health Study and the Nurse's Health Study II cohorts and identified 1007 incident cases of RA [18]. Overall dietary quality as a risk factor for RA was assessed and a dietary index was calculated based on 11 foods (fruits, vegetables, nuts, whole grains, long chain (n-3) fat, polyunsaturated fat...).. Healthy dietary pattern reduced the risk of RA only in women aged 55 and younger (Hazard Ratio highest versus lowest quartile: 0.67; 95\% $\mathrm{Cl}$ : 0.51-0.88; $p=0.002)$. In this study, as well, the protective effect was stronger for rheumatoid 
factor (RF) positive patients. Long chain (n-3) FA, however, were not found to be protective.

In most positive studies a dose-response relationship was noted. As such, the protective effect becomes evident only for moderate to high consumption of fat fish or moderate to high calculated intake levels of omega-3 fatty acid.

\section{Omega-3 fatty acids potential role in preventing auto-antibody formation} in subjects at risk for RA

Another way to investigate the role of omega-3 FA in the etiology of RA was to search for a possible effect in preventing the formation of autoantibodies in subjects at risk for RA, corresponding thus to the pre-RA setting. Several recent studies aimed to determine the association between omega-3 FA and the presence of anti-CCP antibodies in a population at risk for developing RA. In one study [19], FA intake was assessed by a self-reported questionnaire and by measuring the percentage of omega-3 FA in erythrocyte membranes. Reference percentages were based on the findings of a previous study showing that RA patients had lower omega-3 FA levels in erythrocyte membranes than controls [20]. Erythrocyte membrane omega-3 FA measurement is a relevant biomarker of fatty acid exposure as it reveals long term intake [21]. In RA-free individuals who were either HLA-DR4 positive or first degree relatives of RA patients, those who were anti-cyclic citrillunated peptide (anti-CCP) positive had significantly lower FA erythrocyte fraction and declared to consume less omega-3 FA supplements than controls (OR: $0.47,95 \% \mathrm{Cl}$ : $0.24-0.92$ for a S.D. increase in FA levels in erythrocytes, and OR: $0.14, \% 95 \mathrm{Cl}$ : 0.03-0.69, respectively), 
which supports a possible role of omega-3 FA in the prevention of autoantibody development.

When the subjects were stratified based on the positivity of the shared epitope, a negative association between omega-3 FA levels and the presence of autoantibodies (anti-CCP and RF) was observed in shared epitope positive subjects only [22]. Similarly, the use of omega-3 FA supplements was negatively associated with the presence of RF in shared epitope positive subjects but not in shared epitope negative ones [22].

Conversely, in a nested case-control study on incident cases of RA from the European Prospective Investigation into Cancer and Nutrition cohort (EPIC), De Pablo et al. failed to show any association between erythrocyte membrane omega-3 FA amount and the risk of developing RA [23]. Blood samples were collected at inclusion in the cohort (i.e. at the pre-RA phase), and subjects provided information on their diet during the follow-up. Whereas most studies showed a role of omega-3 FA but not omega- 6 FA as protective factors in the development of RA, this study reports a significant negative association between erythrocyte membrane omega- 6 FA linoleic acid (LA) and the risk of RA (highest tertile OR: $0.29 ; 95 \% \mathrm{Cl} 0.12$ to 0.75 ; $\mathrm{p}=0.01$ ). Such an association between low amount of omega- 6 FA and RA was confirmed in the presence of shared epitope. Since this was the first study reporting a possible protective role of omega $6 \mathrm{FA}$ in the development of RA, this finding needs to be confirmed. Although the n-6:n3 FA ratio was not associated with RA incidence in this study, this ratio, frequently used in in cardiovascular research [24] is probably a marker that better reflects the complexity of the system. 


\section{Omega-3 fatty acids and RA incidence in ACPA positive at risk subjects}

After the investigation of the role of omega-3 FA in preventing autoimmunity in individuals at risk of RA, assessing their protective property on the transition from pre-clinical autoimmunity to the established RA, can be considered the next step.

The effect of omega-3 FA in ACPA positive RA-free subjects was reported in a prospective study where 35 anti-CCP3 positive individuals were followed for the determination of inflammatory arthritis [25]. In this study, where 14 incident RA cases were diagnosed after 2.56 years of follow up, 1 S.D. increase in total n-3 FA amount in red blood cells was negatively associated with the incidence of RA (OR 0.09; $95 \% \mathrm{Cl} 0.01$ to $0.85 ; \mathrm{p}=0.03)$. The protective effect was as well observed with docosapentaenoic acid (DPCA), DHA and the association EPA+DHA, measured in erythrocyte membranes. In addition, DPCA \% in red blood cells was inversely associated with the risk of incident RA (Hazard ratio $0.52 ; 95 \% \mathrm{Cl}: 0.27,0.98$; $\mathrm{p}=0.04)$

In summary, despite the conflicting results of dietary studies, the protective role of omega-3 FA against RA development seems to be supported by new data in the setting of pre-RA. Omega-3 FA show a negative association with auto-antibody formation in genetically predisposed individuals, and with RA development in individuals who have already developed biological autoimmunity. Omega-3 FA may then be a clearly identified dietary factor, capable to modulate the course of RA from the pre-clinical phase to the established disease. Such observational data make the rationale for interventional study designed to assess whether omega-3 FA supplementation can actually prevent RA in predisposed individuals. 


\section{References}

[1] Del Gobbo LC, Imamura F, Aslibekyan S, et al. $\omega$-3 Polyunsaturated Fatty Acid Biomarkers and Coronary Heart Disease: Pooling Project of 19 Cohort Studies. JAMA Intern Med 2016;176:1155-66

[2] Racine A, Carbonnel F, Chan SS, et al. Dietary Patterns and Risk of Inflammatory Bowel Disease in Europe: Results from the EPIC Study. Inflamm Bowel Dis 2016;22:345-54

[3] Rodriguez-Cruz M, Cruz-Guzmán ODR, Almeida-Becerril T, et al. Potential therapeutic impact of omega-3 long chain polyunsaturated fatty acids on inflammation markers in Duchenne muscular dystrophy: A double-blind, controlled randomized trial. Clin Nutr 2017; https://doi.org/ 10.1016/j.clnu.2017.09.011 [Epub ahead of print]

[4] Calder PC. Omega-3 polyunsaturated fatty acids and inflammatory processes: nutrition or pharmacology? Br J Clin Pharmacol 2013;75:645-62.

[5] Zhang P, Smith R, Chapkin RS, McMurray DN. Dietary (n-3) polyunsaturated fatty acids modulate murine Th1/Th2 balance toward the Th2 pole by suppression of Th1 development. J Nutr 2005; 135:1745-51

[6] Semerano L,Julia C, Aitisha O, Boissier MC. Nutrition and chronic inflammatory rheumatic disease. Joint Bone Spine 2017;84:547-52

[7] Calder PC, Bond JA, Harvey DJ, Gordon S, Newsholme EA. Uptake and incorporation of saturated and unsaturated fatty acids into macrophage lipids and their effect upon macrophage adhesion and phagocytosis. Biochem J 1990;269:80714

[8] Pompos LJ, Fritsche KL. Antigen-driven murine CD4+ T lymphocyte proliferation and interleukin-2 production are diminished by dietary ( $n-3)$ polyunsaturated fatty acids. J Nutr 2002;132:3293-300

[9] Hughes DA, Pinder AC, Piper Z, Johnson IT, Lund EK. Fish oil supplementation inhibits the expression of major histocompatibility complex class II molecules and adhesion molecules on human monocytes. AM J Clin Nutr 1996:63:267-72 [10] Xue B, Yang Z, Wang X, Shi H. Omega-3 polyunsaturated fatty acids antagonize macrophage inflammation via activation of AMPK/SIRT1 pathway. Plos One 2012;7:e45990 
[11] Singh S, Nair V, Gupta.Linseed oil: an investigation of its anti-arthritic activity in experimental models. Phytother Res 2012;26246-252

[12] lerna M, Kerr A, Scales H, Berge K, Griinari M. Supplementation of diet with krill oil protects against experimental rheumatoid arthritis. BMC Musculoskeletl Disord 2010;11:136

[13] Köhler A, Sarkkinen E, Tapola N, Niskanen T, Bruheim I. Bioavailability of fatty acids from krill oil, krill meal and fish oil in healthy subjects-a randomized, singledose, cross-over trial. Lipids Health D 2015;14:19

[14] Pedersen M, Stripp C, Klarlund M, Olsen SF, Tjønneland A, Frisch M. Diet and risk of rheumatoid arthritis in a prospective cohort. J Rheumatol 2005;32:1249-52 [15] Rosell M, Wesley AM, Rydin K, Klareskog L, Alfredsson L and the EIRA study group. Dietary fish and fish oil and the risk of rheumatoid arthritis. Epidemiology 2009;20:896-901

[16] Shapiro JA, Koepsell TD, Voigt LF, Dugowson CE, Kestin M, Nelson JL. Diet and rheumatoid arthritis in women: a possible protective effect of fish consumption. Epidemiology 1996;7:256-63

[17] Di Giuseppe D, Wallin A, Bottai M, Askling J, Wolk A. Long-term intake of dietary long-chain n-3 polyunsaturated fatty acids and risk of rheumatoid arthritis: a prospective cohort study of women. Ann Rheum Dis 2014;73:1949-53.

[18] Hu Y, Sparks J, Malspeis S, et al. Long-term dietary quality and risk of developing rheumatoid arthritis in women. Ann Rheum Dis 2017;76:1357-1364 [19] Gan RW, Young KA, Zerbe GO, et al. Lower omega 3 fatty acids are associated with the presence of anti -cyclic citrullinated peptide autoantibodies in a population at risk for future rheumatoid arthritis: a nested case-control study. Rheumatology (oxford) 2016;55:367-76

[20]Lee AL, Park Y. The association between n-3 polyunsaturated fatty acid levels in erythrocytes and the risk of rheumatoid arthritis in Korean women. Ann Nutr Metab 2013;63:88-95

[21] Arab L. Biomarkers of fat and fatty acid intake. J Nutr 2003;133:925-32 [22] Gan RW, Demoruelle MK, Deane KD, et al. Omega-3 fatty acids are associated with a lower prevalence of autoantibodies in shared epitope- positive subjects at risk for rheumatoid arthritis. Ann Rheum Dis 2017;76:147-52 
[23] De Pablo P, Romaguera D, Fisk HL, et al. High erythrocyte levels of the n-6 polyunsaturated fatty acid linoleic acid are associated with lower risk of subsequent rheumatoid arthritis in a southern European nested case-control study. Ann Rheum Dis Epub ahead of print: [24 Jan 2018]. doi:10.1136/ annrheumdis-2017-212274 [24] Lee SP, Dart AM, Walker KZ, O'Dea K, Chin-Ducting JP, Skilton MR. Effect of altering dietary n-6:n-3 PUFA ratio on cardiovascular risk measures in patients treated with statins: a pilot study. Br J Nutr 2012;108:1280-5

[25] Gan RW, Bemis EA, Demoruelle MK, et al. The association between omega-3 fatty acid biomarkers and inflammatory arthritis in an anti-citrullinated protein antibody positive population. Rheumatology (oxford) 2017;56:2229-36 [26] Veselinovic M, Vasiljevic D, Vucic V, et al. Clinical benefits of n-3 PUFA and - $^{-}$ linolenic acid in patients with rheumatoid arthritis. Nutrients 2017;9:325 
Figure 1: Omega-3 FA influence on the course of rheumatoid arthritis from pre-clinical phase to established disease. Corresponding references are reported in the Figure.

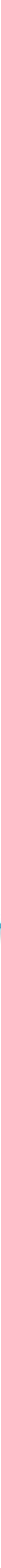

Legends: UA: undifferentiated arthritis, RF: Rheumatoid factor, anti-CCP: anti citrullinated cylic peptide antibodies 